\title{
Post-steroid management of chronic vulvar itching with a topical formula containing natural anti-itching and anti-inflammatory actives
}

This article was published in the following Dove Press journal:

International Journal of Women's Health

18 April 2013

Number of times this article has been viewed

\author{
Francesco Di Pierro' \\ Eleonora Di Maio² \\ Gaetana Di Paola ${ }^{3}$ \\ Raffaele Felice ${ }^{4}$ \\ Filippo Murina ${ }^{4}$ \\ 'Velleja Research, Milan, Italy; \\ ${ }^{2}$ Molinette Hospital, Turin, Italy; ${ }^{3} \mathrm{ASL}$ \\ TO-3/TO-4, Turin, Italy; ${ }^{4}$ Outpatient \\ Department of Vulvar Disease, $V$ Buzzi \\ Hospital-ICP, Milan, Italy
}

\begin{abstract}
Aim: To determine whether use of a topical, nonsteroidal, anti-inflammatory, and anti-itching formula was able to preserve the absence of symptoms, mainly itching and burning, induced by an earlier and relatively short treatment with topical steroids in women diagnosed with vulvar dermatitis or lichen simplex.

Methods: Ninety-six subjects (36 with contact dermatitis, 29 with allergic dermatitis, 31 with lichen simplex) were enrolled in the study. All participants were first treated with topical mometasone furoate (MF) $0.1 \%$. When the symptoms disappeared, they were treated either with Zantogin ${ }^{\circledR}$, a multicomponent topical formula containing anti-inflammatory and anti-itching natural actives, or a control cream for 60 days.

Results: The study demonstrated that, in about $85 \%$ of the participants treated with Zantogin ${ }^{\circledR}$, symptoms disappeared completely, and only $15 \%$ had to resort to MF as needed, with an average use of about three applications per subject (in total). In the placebo group, approximately $90 \%$ of participants had to resort to MF as needed, with an average use per person of more than 16 applications in 60 days.

Conclusion: Our study demonstrates that, following use of a topical steroid, symptoms such as burning and itching can be validly controlled with subsequent and longer therapy with a herbal topical formula, Zantogin ${ }^{\circledR}$, which is able to properly counteract itching and inflammation, prevent symptom relapse, and avoid the typical side effects associated with prolonged use of topical steroids.
\end{abstract}

Keywords: vulvar dermatitis, lichen simplex chronicus, vulvar itching, Zantogin ${ }^{\circledR}$, zanthalene

\section{Introduction}

Vulvar itch is quite common, and, generally, the differential diagnosis is fairly straightforward. In adults, the most frequent cause of itching observed in the vulvar area is dermatitis. In most patients, dermatitis is of the atopic (immune-mediated) type, but a great number of dermatitis disorders are also due to irritant substances or can be described, owing to the whitened and thickened skin appearance with chronic rubbing and scratching, as lichen simplex. ${ }^{1}$ These conditions are quite similar to one another, with itch being the predominant symptom. Burning will occur if the mucosal surface is involved, and fissures can lead to pain and dyspareunia (pain during sexual intercourse). Clinical signs need to be sought carefully as they may be subtle, such as interlabial erythema or small skin splits. Poorly defined erythema (redness), whether with or without scales, may extend onto the pubis and thighs. ${ }^{2}$ The management principles are those applicable to most vulvar diseases and include: patient's education
Correspondence: Francesco Di Pierro Velleja Research, Viale Lunigiana 23,

20I25, Milano, Italy

Tel +393495527663

Fax +390523 5II 894

Email f.dipierro@vellejaresearch.com 
and environmental modification; treatment of any secondary, moderate-to-severe, infection; and, finally, a potent topical steroid, recommended initially once a day (for maximum 1 month) until symptoms disappear. The application rate can be reduced (for example, every second day or three times a week) as symptoms improve. ${ }^{3}$ Because of the chronic course of genital dermatitis and the potential side effects associated with potent topical steroids, alternative, welltolerated therapies are required. ${ }^{4}$ Therefore, we investigated the efficacy and safety of a topical product formulated with natural actives, obtained by extraction processes from botanicals particularly endowed with anti-itching, antiinflammatory, and lenitive properties.

\section{A possible alternative to topical steroids}

The tested product, Zantogin ${ }^{\circledR}$ (Humana Pharma, Milan, Italy; [traded in Italy by PharmExtracta, Pontenure, Italy]), is formulated with 18-beta glycyrrhetinic acid, levomenol, zanthalene (Indena, Milan, Italy), curcumin, tea tree oil, and lactic acid. Eighteen-beta glycyrrhetinic acid is a pentacyclic triterpene obtained from liquorice root (Glycyrrhiza glabra.${ }^{5}$ It has been hypothesized that it is able to inhibit a skin enzyme known as 11-beta-hydroxysteroideidrogenase (11-beta-HSD), which transforms cortisol from the active to the inactive form. Consequently, the inhibition of 11-beta-HSD prolongs cortisol half-life and also its antiinflammatory action. ${ }^{6}$ Levomenol, also known as bisabolol, is an active ingredient characterized by a lenitive action and obtained from chamomile essential oil. ${ }^{7}$ Zanthalene is a $\mathrm{CO}_{2}$ extract obtained from Zanthoxylum bungeanum root standardized to contain alkylamides (30\%), a mixture of alpha-/beta-/gamma-hydroxy sanshool that has been described as being endowed with a strong anti-itching action. ${ }^{8}$ Melaleuca alternifolia oil, also known as tea tree oil, is a well characterized mixture of aromatic compounds with antifungal, antibacterial, and antiviral properties, commonly used in the dermatological and gynecological sectors. ${ }^{9}$ Curcumin is an antioxidant and anti-inflammatory polyphenol extracted from Curcuma longa root. ${ }^{10}$ Lactic acid is an endogenous substance whose function is to control the vaginal $\mathrm{pH}$ value to limit vaginal growth of pathogen strains. ${ }^{11}$ Our work was aimed at defining whether use of a topical, nonsteroidal, anti-inflammatory, and anti-itching formula was able to preserve the absence of symptoms, mainly itching and burning, induced by an earlier and relatively short treatment with topical steroids in women diagnosed with vulvar dermatitis or lichen simplex.

\section{Materials and methods \\ Subjects, enrollment criteria, and first part of the study}

The study was carried out in a routine clinical practice setting, following international guidelines and in line with the principles outlined in the Declaration of Helsinki, ${ }^{12}$ between September 2011 and October 2012, in three Italian outpatient departments in Milan, Turin, and Chivasso, Italy, after informing the local ethical boards. Inclusion criteria were: informed consent and privacy agreement signed and returned; age between 18 and 75 years; total absence of symptoms of infective diseases on enrollment; and diagnosis of dermatitis or lichen simplex with symptoms reported to have lasted for at least 3 months. Exclusion criteria were: refusal to sign the informed consent or privacy agreement; age below 18 or above 75 years; and diagnosis of a vulvar and/or vaginal infective disease. Ninety-six patients were enrolled (36 with contact dermatitis, 29 with allergic dermatitis, and 31 with lichen simplex). At $\mathrm{t}=0$, and after 15 days, all the subjects were assessed in a medical visit for itching, burning, and stretching wounds according to a visual analog scale from 0 to $3(0=$ absent; $1=$ mild; $2=$ strong; $3=$ severe). All subjects were then treated with topical mometasone furoate (MF) $0.1 \%$ for 15 days (one application per day) until the overall score for symptoms ranged between 0 and 1 . If the target score (0-1) was not reached after 15 days of $\mathrm{MF}$, daily use of $\mathrm{MF}$ was continued until the target score was reached.

\section{Products and treatment protocol of the second part of the study}

After the first check (15th day), all subjects were randomized by an independent investigator, using a computer-generated random number table, to either of two groups: one receiving a topical treatment for 60 days, with one application per day of Zantogin ${ }^{\circledR}$ cream, a multicomponent formula containing 18-beta glycyrrhetinic acid phytosome (1.5\%), levomenol $(0.1 \%)$, zanthalene $(0.5 \%)$, curcumin phytosome $(0.25 \%)$, tea tree oil $(0.05 \%)$, and lactic acid $(0.2 \%)$; and one treated with a control cream (placebo) containing the same doses of tea tree oil and lactic acid as the test cream but totally devoid of the other four actives. For this reason, the subjects of both groups (48 per group) thought they were being treated with an active cream. According to the earlier diagnosis, the Zantogin ${ }^{\circledR}$ group comprised 18 subjects with contact dermatitis, 15 with allergic dermatitis, and 15 with lichen simplex; the placebo 
group comprised 18 with contact dermatitis, 14 with allergic dermatitis, and 16 with lichen simplex. During the 60 days of the second phase of the study, medical checks occurred every 10 days, but data were collected and recorded, as planned, only at the end of the study (after 60 days). During the 60 days of the second phase of the study all subjects were allowed to use topical MF as required.

\section{End points, time, and methods of scoring}

Primary end points were the evaluation of the absence of symptoms and need to use MF. Tolerability (scored as very poor, poor, fair, good, or excellent) and side effects were also assessed. All data were collected by using a questionnaire in which all subject demographic data were reported along with a table in which the physician recorded the score in relationship to the symptom. The scores recorded at $t=0$, at $t=15$, at $t=60$ was always the result of the integration of what the subject reported from her point of view in terms of itching and burning and what was visible by the physician both globally, from a gynecological point of view, and in particular, as regards to stretching of wounds. In the same way, tolerability and side effects were recorded. All subjects were then issued a questionnaire, for in-house use, by which to record every day their application of MF.

\section{Statistical analysis}

The statistical analysis used to check data variations (itching, burning, stretching of wounds, and use of MF as needed) was between/within-subject analysis of variance (ANOVA) applied to two factors: treatment and period.

\section{Results}

Because of the chronic courses of both vulvar dermatitis and lichen simplex, and due to the potential side effects associated with topical steroids commonly used to stop itching and burning, alternative, well-tolerated therapies should be considered. To this end, we investigated the efficacy and safety of a topical product formulated with natural actives characterized by anti-itching, anti-inflammatory, and lenitive properties. The efficacy of the test product, Zantogin ${ }^{\circledR}$ (18-beta glycyrrhetinic acid, levomenol, zanthalene, curcumin, tea tree oil, and lactic acid), was compared with a placebo treatment (only containing tea tree oil and lactic acid). The baseline characteristics of the 96 enrolled subjects are reported in Table 1. As shown, there was no difference in terms of age or duration of symptoms. The same also applied to the overall score at $\mathrm{t}=0$, when all subjects began
Table I Personal and baseline characteristics of the subjects (48 per group)

\begin{tabular}{lcc}
\hline Variable & Zantogin $^{\circledR}$ & Placebo \\
\hline Age (years) & $57.9 \pm 13.9$ & $56.2 \pm 14.6$ \\
Duration of symptoms (months) & $4.5 \pm 1.5$ & $4.0 \pm 1.5$ \\
Score $^{\mathrm{a}}$ at $\mathrm{t}=$ O (before MF) & $7.9 \pm 1.8$ & $7.6 \pm 1.7$ \\
Score $^{\mathrm{a}}$ following MF & $0.4 \pm 0.2^{*}$ & $0.3 \pm 0.2^{*}$ \\
\hline
\end{tabular}

Notes: Data are presented as mean value \pm standard deviation. ${ }^{\text {aAccording }}$ to a visual analog scale from 0 to 3 ( 0 = absent; $I$ = mild; 2 = strong; 3 = severe); a global score was given after assessing for itching, burning, and stretching of wounds. $* \mathrm{P}<0.01$ versus score at $\mathrm{t}=0$.

Abbreviation: MF, mometasone furoate.

MF treatment, and the score at the end of the MF protocol. Of note, the 15-day MF protocol was shown to be very effective in 91 out of 96 subjects; however, after 15 days of treatment, five participants out of 96 did not have a global score between 0 and 1, as planned in the study protocol. For this reason, MF treatment was continued in these five people for another 10 days, which allowed the global score to drop between 0 and 1 and, after assignment to placebo $(n=2)$ or to Zantogin ${ }^{\circledR}(n=3)$, they were continued to the second phase of the study as reported in the "Materials and methods" section.

After evaluation of symptoms and ascertaining that the overall score had decreased below 1 according to the study protocol, all 96 subjects were treated with either Zantogin ${ }^{\circledR}$ or a placebo formula. Ninety-three of the 96 subjects completed the study, while three dropped out. Since all subjects had the possibility of using MF as needed, there were no differences in terms of total score with either treatment, Zantogin ${ }^{\circledR}$ or placebo (Table 2). Clear differences are observable between the two groups, however, when considering the number of subjects using MF or the number of total MF applications. Only seven of the 46 subjects $(15.2 \%)$ of the Zantogin ${ }^{\circledR}$ group resorted to MF as needed against 42 of the 47 patients $(89.4 \%)$ of the placebo group. When considering the total

Table 2 Clinical score after 60-day treatment, as evaluated in subjects who completed the study, number of subjects using MF as needed, and number of total application of MF

\begin{tabular}{lllll}
\hline Group & $\mathbf{n}$ & $\begin{array}{l}\text { Score }^{\mathrm{a}} \text { at } \mathbf{t}=\mathbf{6 0} \\
\mathbf{( M \pm} \pm \mathbf{S D})\end{array}$ & $\begin{array}{l}\text { Subjects } \\
\text { using } \mathbf{M F} \\
(\mathbf{n})\end{array}$ & $\begin{array}{l}\text { Total MF } \\
\text { applications } \\
(\mathbf{n})\end{array}$ \\
\hline Zantogin $^{\circledR}$ & 46 & $0.5 \pm 0.2$ & $7^{*}$ & $23^{*}$ \\
Placebo & 47 & $0.8 \pm 0.4$ & 42 & 682 \\
\hline
\end{tabular}

Notes: ${ }^{a}$ According to a visual analog scale from 0 to $3(0=$ absent; $1=$ mild; 2 = strong; 3 = severe); a global score was given after assessing for itching, burning, and stretching of wounds. $* P<0.01$ versus placebo.

Abbreviations: M, mean; MF, mometasone furoate; SD, standard deviation. 
number of MF applications, the differences become even more important: 23 applications in the Zantogin ${ }^{\circledR}$ group, with an average of about three applications overall per subject, against 682 in the placebo group, with an average of about 16 applications over 60 days per subject.

In terms of tolerability, Zantogin ${ }^{\circledR}$ showed good tolerability with only two dropouts because of burning induced in the first 5 treatment days, possibly due to sensitivity reactions. Comparable tolerability, side effects, and dropouts were observed in the placebo group (Table 3 ).

\section{Discussion}

Vulvar itching due to contact dermatitis, allergic dermatitis, or lichen simplex is one of the most common symptoms in vulvar disorders. Daily topical application of steroidal formulas is very frequent and effective. However, the chronic aspects of both symptoms (itching) and diseases (dermatitis and lichen simplex), and the side effects commonly induced by topical application of steroids, suggest the need for alternative and well-tolerated therapies. To this end, we investigated the efficacy and tolerability of a topical formula $\left(\right.$ Zantogin $\left.^{\circledR}\right)$ prepared by using herbal actives endowed with anti-inflammatory, anti-itching, and lenitive properties. The formula was tested in patients following topical application of MF due to a diagnosis of vulvar dermatitis or lichen simplex. Zantogin ${ }^{\circledR}$, containing 18-beta glycyrrhetinic acid, levomenol, zanthalene, curcumin, tea tree oil, and lactic acid, was compared with a placebo formula containing only those actives devoid of any direct anti-inflammatory, anti-itching, and lenitive properties but present in the Zantogin ${ }^{\circledR}$ formula as natural preservatives and $\mathrm{pH}$ balancers: tea tree oil and lactic acid. The results demonstrated that, in about 85\% (39 of 46) of the subjects treated with Zantogin ${ }^{\circledR}$, symptoms disappeared completely, and that only $15 \%$ (seven of 46) required the steroidal application as needed, with an average use of about three applications ( 3 days out of 60) per subject. These results appear to be quite relevant if compared with those obtained in the placebo group, where about $89 \%$ (42 of 47)

Table 3 Tolerability, side effects, and dropouts

\begin{tabular}{llll}
\hline Group & Tolerability & Dropouts & Side effects \\
\hline Zantogin $^{\circledR}$ & Excellent $(\mathrm{n}=26)$ & $\mathrm{n}=2$ & Burning $(\mathrm{n}=2)$ \\
& $\begin{array}{l}\text { Good }(\mathrm{n}=20) \\
\text { Very poor }(\mathrm{n}=2)\end{array}$ & & \\
Placebo & Excellent $(\mathrm{n}=36)$ & $\mathrm{n}=1$ & Burning $(\mathrm{n}=\mathrm{I})$ \\
& Good $(\mathrm{n}=10)$ & & \\
& Very poor $(\mathrm{n}=1)$ & & \\
\hline
\end{tabular}

of the subjects had to resort to MF, with an average use per person of more than 16 applications in 60 days. (about two applications a week). The strong efficacy of the Zantogin ${ }^{\circledR}$ preparation can be explained, at least in part, by analyzing the biological properties of its actives. One of these (Zanthalene) appears to be quite important. Its alkylamides reduce skin sensitivity. Their mechanism of action can be highlighted by previous data ${ }^{13}$ on the nerve-muscle in vitro tests in which the $Z$. bungeanum extract, titrated in alkylamides, showed a marked and transitory effect on the neuromuscular synaptic transmission (increase in miniature potential frequency and occurrence of spontaneous endplate potentials). The previous results suggest that this activation may rapidly lead to neurotransmitter depletion and, consequently, to subsequent synaptic transmission block. The resulting data also suggest that the extract action is mediated by voltage-dependent $\mathrm{Na}^{+}$ channels, either through a direct action of the substance on the channels or through a general depolarizing action of the extract. On the basis of the results of the membrane potentials, the latter hypothesis seems to be the less probable. Finally, the results obtained in tests performed with d-tubocurarine exclude a direct action on muscles. The synaptic transmission block thus seems to be responsible for the marked anti-itching and anesthetic-like actions observed. Another important issue concerning the efficacy observed with the Zantogin ${ }^{\circledR}$ preparation is the use of the phytosome forms for two actives of its formula. Both 18-beta glycyrrhetinic acid and curcumin were used in a particular form in which they were chemically linked with distearoylphosphatidylcholine from soy. Such a link provides better penetrability of the actives into the different skin layers and, therefore, very clear dermatological activity. ${ }^{14}$ Our study demonstrates that use of topical steroids, for a relatively short period of time, in women with significant vulvar itching linked to a diagnosis of vulvar dermatitis or lichen simplex, leads to excellent control of symptoms. These can be validly controlled with a subsequent and longer therapy with a herbal topical formula, Zantogin ${ }^{\circledR}$, capable of properly counteracting itch and inflammation, preserving complete absence of symptoms and avoiding the typical side effects associated with prolonged use of topical steroids. New studies need to be undertaken to check if Zantogin ${ }^{\circledR}$ could reduce vulvar symptomatology without topical steroid pretreatment.

\section{Disclosure}

FDP is the main formulator of Zantogin ${ }^{\circledR}$. The other authors report no conflicts of interest in this work. 


\section{References}

1. Fischer GO. The commonest causes of symptomatic vulvar disease: a dermatologist's perspective. Australas J Dermatol. 1996;37:12-18.

2. Thorstensen KA, Birenbaum DL. Recognition and management of vulvar dermatologic conditions: lichen sclerosus, lichen planus, and lichen simplex chronicus. J Midwifery Womens Health. 2012;57(3):260-275.

3. Kelekci KH, Adamhasan F, Gencdal S, Sayar H, Kelekci S. The impact of the latest classification system of benign vulvar diseases on the management of women with chronic vulvar pruritus. Indian J Dermatol Venereol Leprol. 2011;77(3):294-299.

4. Johnson E, Groben P, Eanes A, Iyer P, Ugoeke J, Zolnoun D. Vulvar skin atrophy induced by topical glucocorticoids. J Midwifery Womens Health. 2012;57(3):296-299.

5. Asl MN, Hosseinzadeh H. Review of pharmacological effects of Glycyrrhiza sp. and its bioactive compounds. Phytother Res. 2008; 22(6):709-724.

6. Hennebold JD, Daynes RA. Inhibition of skin 11beta-hydroxysteroid dehydrogenase activity in vivo potentiates the anti-inflammatory actions of glucocorticoids. Arch Dermatol Res. 1998;290(8):413-419.

7. McKay DL, Blumberg JB. A review of the bioactivity and potential health benefits of chamomile tea (Matricaria recutita L.). Phytother Res. 2006; 20(7):519-530.
8. Artaria C, Maramaldi G, Bonfigli A, Rigano L, Appendino G. Lifting properties of the alkamide fraction from the fruit husks of Zanthoxylum bungeanum. Int J Cosmet Sci. 2011;33(4):328-333.

9. Carson CF, Hammer KA, Riley TV. Melaleuca alternifolia (Tea Tree) oil: a review of antimicrobial and other medicinal properties. Clin Microbiol Rev. 2006;19(1):50-62.

10. Jurenka JS. Anti-inflammatory properties of curcumin, a major constituent of Curcuma longa: a review of pre-clinical and clinical researches. Altern Med Rev. 2009;14(2):141-153.

11. Dover SE, Aroutcheva AA, Faro S, Chikindas ML. Safety study of an antimicrobial peptide lactocin 160, produced by the vaginal Lactobacillus rhamnosus. Infect Dis Obstet Gynecol. 2007;2007:78248.

12. Levine RJ. Some recent developments in the international guidelines on the ethics of research involving human subjects. Ann NY Acad Sci. 2000;918:170-178.

13. Di Pierro F, Callegari A, Catacchio V, Attolico M, Alfonso R. Derivati Botanici nel trattamento delle vaginiti atrofiche. [Botanical derivatives in athrophic vaginitis treatment]. Cosmetic Technology 2008;11(6):23-27. Italian.

14. Bombardelli E. Phytosome: new cosmetic delivery system. Boll Chim Farm. 1991;130(11):431-438.
International Journal of Women's Health

\section{Publish your work in this journal}

The International Journal of Women's Health is an international, peerreviewed open-access journal publishing original research, reports, editorials, reviews and commentaries on all aspects of women's healthcare including gynecology, obstetrics, and breast cancer. The manuscript management system is completely online and includes

\section{Dovepress}

a very quick and fair peer-review system, which is all easy to use. Visit http://www.dovepress.com/testimonials.php to read real quotes from published authors.

\footnotetext{
Submit your manuscript here: http://www.dovepress.com/international-journal-of-womens-health-journal
} 